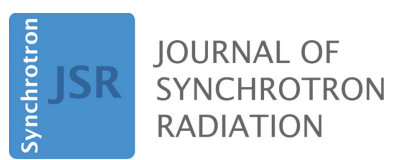

ISSN 1600-5775

Received 2 June 2017

Accepted 27 October 2017

Edited by Y. Amemiya, University of Tokyo, Japan

Keywords: free-electron laser; soft X-ray; beamline instrumentation.
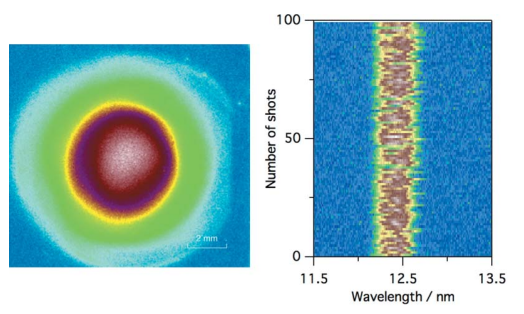

OPEN $\odot$ ACCESS

\section{A soft X-ray free-electron laser beamline at SACLA: the light source, photon beamline and experimental station}

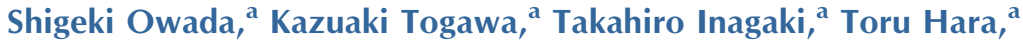 \\ Takashi Tanaka, ${ }^{\text {a }}$ Yasumasa Joti, ${ }^{\mathrm{b}}$ Takahisa Koyama, ${ }^{\text {b }}$ Kyo Nakajima, \\ Haruhiko Ohashi, ${ }^{\mathrm{b}}$ Yasunori Senba, ${ }^{\mathrm{b}}$ Tadashi Togashi, ${ }^{\mathrm{b}}$ Kensuke Tono, \\ Mitsuhiro Yamaga, ${ }^{\mathrm{b}}$ Hirokatsu Yumoto, ${ }^{\mathrm{b}}$ Makina Yabashi, ${ }^{\mathrm{a} *}$ Hitoshi Tanaka ${ }^{\mathrm{a} *}$ and \\ Tetsuya Ishikawa ${ }^{a}$
}

aRIKEN SPring-8 Center, Sayo-cho, Sayo-gun 679-5148, Japan, and bJapan Synchrotron Radiation Research Institute,
Sayo-cho, Sayo-gun 679-5948, Japan. ${ }^{*}$ Correspondence e-mail: yabashi@spring8.or.jp, tanaka@spring8.or.jp

The design and performance of a soft X-ray free-electron laser (FEL) beamline of the SPring- 8 Compact free-electron LAser (SACLA) are described. The SPring-8 Compact SASE Source test accelerator, a prototype machine of SACLA, was relocated to the SACLA undulator hall for dedicated use for the soft X-ray FEL beamline. Since the accelerator is operated independently of the SACLA main linac that drives the two hard X-ray beamlines, it is possible to produce both soft and hard X-ray FEL simultaneously. The FEL pulse energy reached $110 \mu \mathrm{J}$ at a wavelength of $12.4 \mathrm{~nm}$ (i.e. photon energy of $100 \mathrm{eV}$ ) with an electron beam energy of $780 \mathrm{MeV}$.

\section{Introduction}

The advent of X-ray free-electron lasers (XFELs) has stimulated marked progress in various scientific fields, such as ultrafast chemistry (Moshammer et al., 2007; Erk et al., 2014), nonlinear X-ray optics (Rohringer et al., 2012; Glover et al., 2012; Bencivenga et al., 2015) and structural biology (Boutet et al., 2012; Nango et al., 2016). The first FEL operation in the extreme ultraviolet (EUV) region was achieved by the TESLA Test Facility (TTF; Andruszkow et al., 2000) at DESY, Germany, in 2000, which was later renamed FLASH (Ackermann et al., 2007). The FEL wavelengths were shortened to the angstrom region by the Linac Coherent Light Source (LCLS) (Emma et al., 2010) at the SLAC National Accelerator Laboratory (SLAC), USA, in 2009. A soft X-ray FEL based on a high-harmonic high-gain (HGHG) seeding scheme was also in operation at FERMI in Elettra, Italy (Allaria et al., 2012).

The SPring-8 Ångstrom Compact free-electron LAser (SACLA), constructed in Harima, Japan, achieved first lasing at $10 \mathrm{keV}$ in June 2011 and started operation for users in March 2012 with two beamlines: BL3 for a hard X-ray FEL, which is capable of generating the shortest wavelength FEL below $0.8 \AA$, and BL1 for wide range spontaneous emission (Ishikawa et al., 2012; Yabashi et al., 2015). Based on unique capabilities and continuous upgrades (Hara et al., 2013; Mimura et al., 2014; Katayama et al., 2016), BL3 has been providing research opportunities in various fields of science (Shwartz et al., 2014; Tamasaku et al., 2014; Yoneda et al., 2014, 2015; Kim et al., 2015; Inoue et al., 2016). The success of BL3 
and users' demands for higher availability led us to construct the second XFEL beamline (BL2), which has been operating since April 2015. At the same time, a much smaller number of research proposals applied for BL1, mainly because only spontaneous radiation was available with one undulator unit of $4.5 \mathrm{~m}$ length. In addition, the operation of BL1 limited the availability of BL2 and BL3, because the SACLA main linac had been commonly employed to all beamlines.

However, requirements for utilizing soft X-ray FELs have been continuously increasing. To meet the demands, we commenced a project to upgrade BL1 in 2014. A marked feature was to re-employ the SCSS test accelerator (SCSS; Shintake et al., 2008) as an electron beam driver dedicated to BL1, independent of the SACLA main linac operating for BL2 and BL3; the SCSS was relocated to a $90 \mathrm{~m}$-long empty space on the upstream side of the dog-leg section of BL1 in the SACLA undulator hall, which can accommodate high-gradient C-band accelerator units to generate a several hundred $\mathrm{MeV}$ to $\sim 1 \mathrm{GeV}$ electron beam.

The original SCSS machine was constructed as a prototype of SACLA for evaluation of the feasibility of a compact XFEL concept as well as development of experimental techniques with FELs. The construction of the SCSS with a $250 \mathrm{MeV}$ linac and two undulators was completed in 2005. The first lasing at a wavelength of $\lambda=49 \mathrm{~nm}$ was achieved in 2006, followed by user operation in 2007 (Sato et al., 2008). Furthermore, the first high-harmonic-generation (HHG) seeded FELs in the ultraviolet (UV) and EUV region were demonstrated (Lambert et al., 2008; Togashi et al., 2011; Tomizawa et al., 2015). After these achievements and successful operation of SACLA, the SCSS was decommissioned in 2013.

In 2014, we started relocation of the SCSS to the SACLA undulator hall with the addition of one C-band accelerator unit (i.e. three units in total) to increase the electron beam energy to $\sim 500 \mathrm{MeV}$. After the RF conditioning, we started the commissioning of this machine, which we call the SCSS+, in September 2015, and readily observed the first lasing at $\lambda=$ $33.7 \mathrm{~nm}$ (i.e. photon energy $h v=36.8 \mathrm{eV}$ ) on 7 October 2015. We finished the commissioning of the photon beamline and the construction of the experimental station in June 2016, followed by the first user operation in July 2016.

This paper reports the design and performance of the soft X-ray FEL beamline BL1 at SACLA. The design of the light source, the beamline optics and beam diagnostics, and the experimental station will be described in $\$ 2$. Characteristics of soft X-ray FEL light are then described in $\$ 3$ and finally we will show the future perspective in $\S 4$.
Table 1

Machine parameters of the SCSS+ accelerator.

\begin{tabular}{ll}
\hline Parameter & Present value \\
\hline Electron beam & \\
Beam energy & $800 \mathrm{MeV}$ (maximum) \\
Bunch charge & $0.2-0.3 \mathrm{nC}$ \\
Peak current & $300 \mathrm{~A}$ (simulation) \\
Energy spread (projected) & $0.6 \%$ (FWHM) \\
Normalized emittance (projected) & $3 \mathrm{~mm} \mathrm{mrad}$ \\
Repetition rate & $60 \mathrm{~Hz}$ \\
Undulator & $18 \mathrm{~mm}$ \\
Periodic length & 3 \\
Number of undulator modules & 777 \\
Total number of period & 2.1 \\
Maximum $K$ & $3.8 \mathrm{~mm}$ \\
Minimum gap & $6 \mathrm{~m} / 4 \mathrm{~m}$ \\
Averaged betatron function, $\beta x / \beta y$ & \\
\hline
\end{tabular}

\section{Design}

\subsection{Light source}

The layout of the light source apparatus SCSS+ and the machine parameters are shown in Fig. 1 and Table 1, respectively. The electron accelerator and the undulator are wholly located beside the XFEL beamlines BL2 and BL3 in the SACLA undulator hall. The distance from the electron gun to the undulator hall end is $230 \mathrm{~m}$, including a $140 \mathrm{~m}$ drift space for future energy upgrade.

The electron gun with a $\mathrm{CeB}_{6}$ thermionic cathode launches a microsecond electron pulse with an energy of $500 \mathrm{keV}$ and a peak current of $1 \mathrm{~A}$ at a repetition rate of $60 \mathrm{~Hz}$ (Togawa et al., 2007). A central part of $1 \mathrm{~ns}$ is cut out from the microsecond pulse using a beam chopper followed by the buncher section that consists of a $238 \mathrm{MHz}$ pre-buncher and a $476 \mathrm{MHz}$ booster (Shintake et al., 2009). The beam is compressed down to about $10 \mathrm{ps}$ due to velocity modulation by these multifrequency cavities and bunched after the flight in the drift space. The S-band linac accelerates the bunched beam to $49 \mathrm{MeV}$ while providing a negative energy chirp over the whole bunch to further compress it by the following first bunch compressor (BC1). An energy filter located at the dispersive section of the $\mathrm{BC} 1$ removes longitudinal head and tail parts of the bunch; the charge of $\sim 0.2 \mathrm{nC}$ is sent downstream. The normalized projected emittance of the bunch was measured to be $3 \mathrm{~mm}$ mrad by the quadrupole-magnet-scan method at the BC1 exit.

Five C-band accelerator units are used for main beam acceleration (Inagaki et al., 2014; Sakurai et al., 2017). Each unit has two $1.8 \mathrm{~m}$ accelerating structures, whose maximum

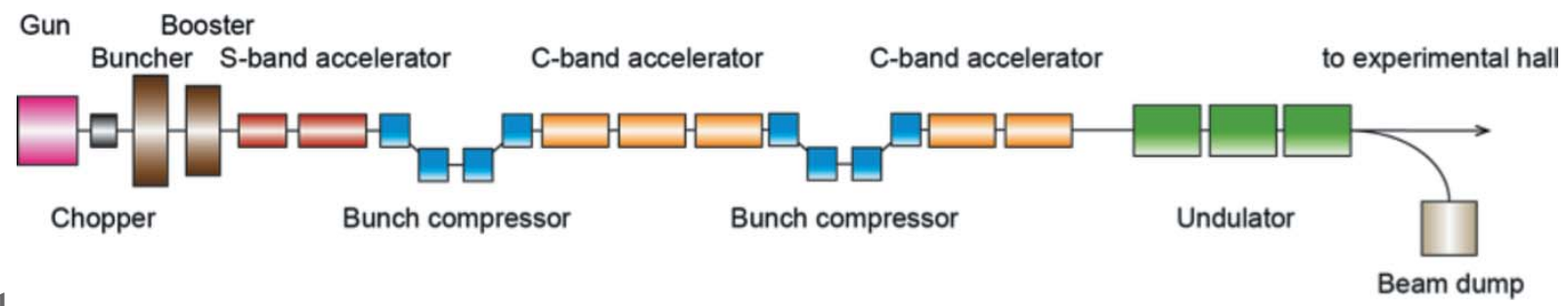

Figure 1

Layout of the SCSS+ accelerator. 


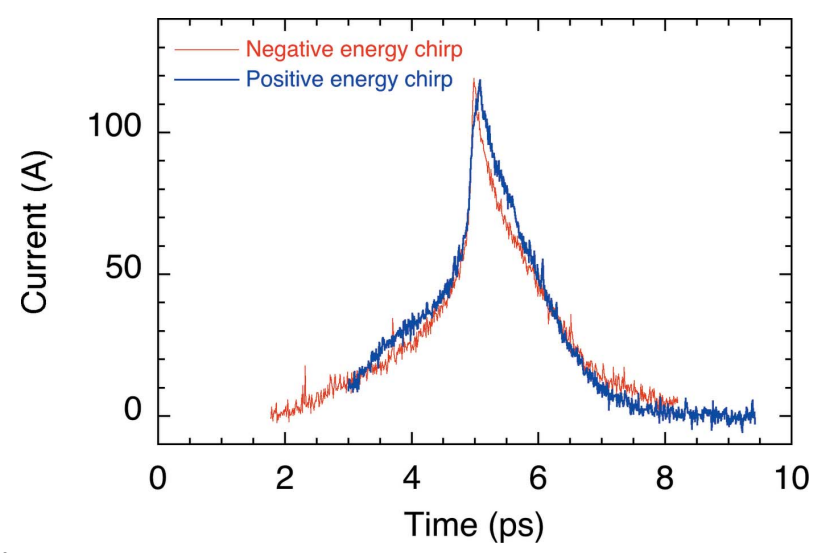

Figure 2

Longitudinal current profiles of the electron beam at the exit of the first bunch compressor measured by means of an RF zero-phasing method. Two profiles obtained by providing positive and negative energy chirps coincide with each other.

energy gain is more than $150 \mathrm{MeV}$. The first three units accelerate the bunch at an off-crest phase for the compression at the second bunch compressor (BC2); the other two units accelerate the bunch at the on-crest phase to the final beam energy. The final energy can be tuned in the range between 300 and $800 \mathrm{MeV}$ to control FEL wavelengths over a wide range.

The longitudinal bunch structure after the $\mathrm{BC} 1$ was measured by an RF zero-phasing method (Wang et al., 1998). A linear energy chirp was added along the bunch by the zerocrossing phase of the third C-band unit. Then the energy profile, which corresponds to the time structure of the bunch, was analysed at the dispersive section of $\mathrm{BC} 2$. The peak current and the width of the bunch were measured to be $\sim 120 \mathrm{~A}$ and $0.8 \mathrm{ps}$ full width at half-maximum (FWHM), respectively, as shown in Fig. 2. Since there is no diagnostic apparatus such as an RF deflector after the $\mathrm{BC} 2$, an exact bunch profile at the undulator section has not been measured so far. Based on the particle tracking simulation using PARMELA and ELEGANT codes, we estimated that a peak current of $\sim 300 \mathrm{~A}$ and a bunch width of $0.5 \mathrm{ps}$ (FWHM) are achieved after the final compression. The normalized slice emittance of the bunch was analysed to be $1 \mathrm{~mm}$ mrad.

Three in-vacuum undulator units, whose magnet design is the same as the SACLA undulator, are used to generate soft
X-ray FEL light (Tanaka et al., 2008). The undulator gap range is from 3.8 to $20 \mathrm{~mm}$; the maximum deflection parameter $(K$ value) is 2.1. The periodic length of the permanent magnet and the total number of periods of the three units are $18 \mathrm{~mm}$ and 777 , respectively. The FEL radiation wavelength can be varied between 8 and $50 \mathrm{~nm}$ by tuning the electron beam energy and the undulator $K$-value. The characteristics of FEL are described in $\S 3$.

An electron-beam-based alignment was performed during the undulator commissioning. The electron beam orbits were measured at different beam energies. In order to achieve a dispersion-free condition in the undulator section, the strengths of the steering coils located in front of each undulator unit were determined so that the beam orbit is kept constant while changing the beam energy. After that, the injection position and angle of the beam into the undulator section, the RF phases of the accelerator and the strengths of the focusing magnets were tuned to maximize the FEL pulse energy.

\subsection{Beamline}

2.2.1. Layout of beamline. Fig. 3 shows the top view of the photon beamline. It consists of the front-end section in the SACLA undulator hall, the transport channel in the optical hutch $(\mathrm{OH})$ and the experimental station $4 \mathrm{a}(\mathrm{EH} 4 \mathrm{a})$ in the SACLA experimental hall. The beamline optics and diagnostics instruments are mainly installed in the $\mathrm{OH}$. Because of the high absorption cross section in the soft X-ray region, the beamline is windowless, and kept at ultra-high vacuum (UHV, $<10^{-6} \mathrm{~Pa}$ ).

2.2.2. Beamline optics. The optical system of BL1 consists of a plane mirror and gas/foil attenuators. For the plane mirror, we utilize a $400 \mathrm{~mm}$-long Si substrate partially coated with carbon, which provides high reflectivity in a wavelength range longer than the carbon $K$-edge of $4.4 \mathrm{~nm}$ at a glancing angle of $1.5^{\circ}$. The plane mirror is installed at a distance from the exit of the last undulator of $L=45 \mathrm{~m}$.

The gas and foil attenuators installed in the $\mathrm{OH}$ are used to control the FEL pulse energy. The foil attenuators, which consist of $\mathrm{Al}, \mathrm{Si}, \mathrm{Sn}, \mathrm{Ti}$ and $\mathrm{Zr}$ (see Table 2 for details), can be combined with the gas attenuator (GAT) at $L=50 \mathrm{~m}$ to select the fundamental or the higher harmonics. For GAT, a $2.6 \mathrm{~m}$ long chamber is filled with $\mathrm{N}_{2}$ with a pressure of up to $100 \mathrm{~Pa}$.

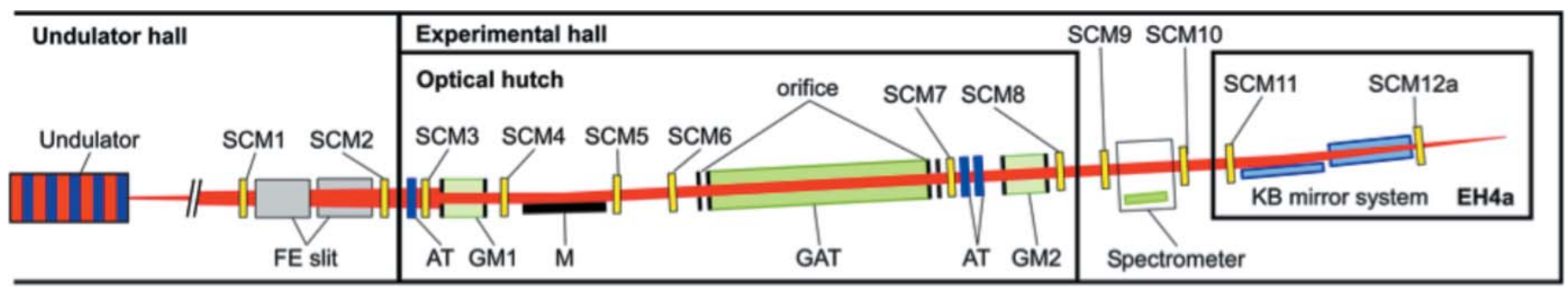

Figure 3

Schematic drawing of BL1 (top view). FE slit: front-end slit; SCM: screen monitor system; AT: foil attenuator; GAT: gas attenuator; M: plane mirror (partial C coating on Si substrate); GM: gas intensity monitor. 
Table 2

Specification of the foil attenuators.

\begin{tabular}{ll}
\hline Attenuator & Thickness \\
\hline $\mathrm{Al}$ & $0.1,0.2,0.3,0.4,0.5 \mu \mathrm{m}$ \\
$\mathrm{Si}$ & $0.1,0.3 \mu \mathrm{m}$ \\
$\mathrm{Sn}$ & $0.1,0.2,0.5 \mu \mathrm{m}$ \\
$\mathrm{Ti}$ & $0.1 \mu \mathrm{m}$ \\
$\mathrm{Zr}$ & $0.1,0.2,0.5,1.0,2.0 \mu \mathrm{m}$
\end{tabular}

To keep the UHV condition through the beam path, differential pumping chambers with $6 \mathrm{~mm}$-diameter orifices are attached to both sides of the GAT.

2.2.3. Beam diagnostics. Photon diagnostic systems such as photodiodes (SXUV-300C, OptDiode), Ce:YAG screens (Konoshima Chemical) and a spectrometer that combines a variable-line-spacing plane grating (30-002, Shimadzu) with a phosphor-coupled microchannel plate (F2224, Hamamatsu Photonics), are installed in the transport channel of BL1. The spectrometer is calibrated using the absorption spectrum of aluminium $L_{2,3}$-edge and the emission spectrum of the $\mathrm{He}^{\mathrm{II}}$ $2 p$ to $1 s$ and $3 p$ to $1 s$ transition.

Since the photon beam parameters can fluctuate shot-byshot, nondestructive diagnostics are important for analysis of experimental data. For nondestructive diagnostics of the pulse energy, we developed a gas intensity monitor (GM), which works as an ion chamber to detect photoionization of rare gas with an electron multiplier (R2362, Hamamatsu Photonics). In the present study, the chamber of the GM was filled with Ar at a pressure of $\sim 10^{-4} \mathrm{~Pa}$. The signal intensity was converted to the ion current and finally to the pulse energy through calibration with a calorimeter (Tanaka et al., 2015). We installed two GMs in the OH: GM1 at $L=43 \mathrm{~m}$ is used for measuring the pulse energy without attenuators, while GM2 at $L=60 \mathrm{~m}$ measures the pulse energy after the attenuators. Similar to GAT, we attached differential pumping chambers with $6 \mathrm{~mm}$ diameter orifices to both sides of the GMs. The orifice size was determined so as to transmit more than $95 \%$ of the incident

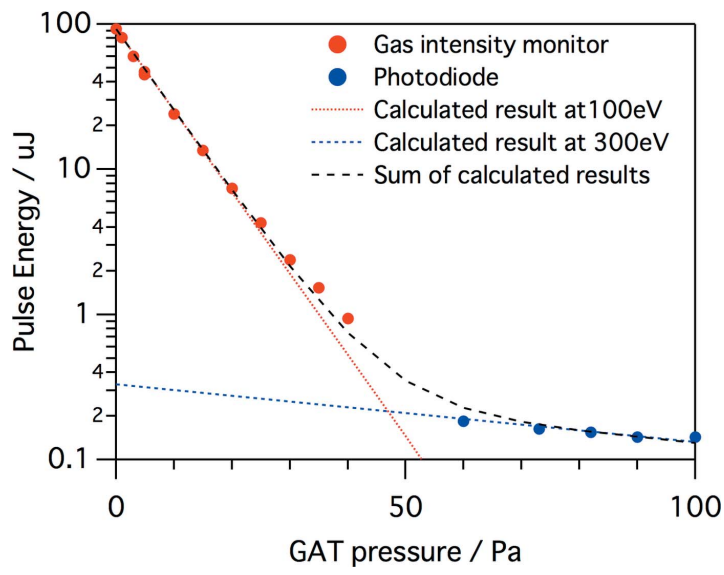

Figure 4

Dependence of FEL pulse energy on $\mathrm{N}_{2}$ pressure measured with GMs and the silicon photodiode. The red dashed line shows a calculated result by the Henke's cross-section data at $\lambda=12.4 \mathrm{~nm}$, while the blue dashed line is that given at $\lambda=4.1 \mathrm{~nm}$. The black dashed line shows the summation of the two calculated results.
Table 3

Design of the KB mirrors.

\begin{tabular}{lll}
\hline & Horizontal & Vertical \\
\hline Mirror size & $600 \times 50 \times 50 \mathrm{~mm}$ & $600 \times 50 \times 50 \mathrm{~mm}$ \\
Coating & Carbon & Carbon \\
Glancing angle & $1.5^{\circ}$ & $1.5^{\circ}$ \\
Spatial acceptance & $15.1 \mathrm{~mm}$ & $15.1 \mathrm{~mm}$ \\
Focal length & $2.65 \mathrm{~m}$ & $2.00 \mathrm{~m}$ \\
Geometrical spot size & $\sim 3 \mu \mathrm{m} @ \lambda=10 \mathrm{~nm}$ & $\sim 3 \mu \mathrm{m} @ \lambda=10 \mathrm{~nm}$ \\
\hline
\end{tabular}

pulse energy ( $4 \sigma$ width of the beam size) at $\lambda=12.4 \mathrm{~nm}$ based on the simulation result using SIMPLEX (Tanaka, 2015).

By combining these tools, we measured the FEL transmittance at a wavelength $\lambda=12.4 \mathrm{~nm}$ while changing the gas pressure of the GAT, as shown in Fig. 4. We used two GMs for the lower attenuation region from 0 to $40 \mathrm{~Pa}$, while we combined GM1 and the photodiode for the higher attenuation above $60 \mathrm{~Pa}$. The dependence between 0 and $30 \mathrm{~Pa}$ agrees with those calculated with the Henke's cross-section data (Henke et al., 1993) at $\lambda=12.4 \mathrm{~nm}$, while the small mismatch between 30 and $40 \mathrm{~Pa}$ is explained by the contribution of the third-order harmonics of $\lambda=4.1 \mathrm{~nm}$. The measured curve between 60 and $100 \mathrm{~Pa}$ is simply reproduced with the calculated result at $\lambda=4.1 \mathrm{~nm}$, which indicates that the third-order harmonics is dominant at the gas attenuator pressure higher than $60 \mathrm{~Pa}$. The contribution of the third harmonics without attenuation is estimated to be $\sim 0.3 \%$, as seen in Fig. 4 .

\subsection{Experimental station}

We built one experimental station, called EH4a, at the end of the beamline. A Kirkpatrick-Baez (KB) focusing mirror system and a synchronized optical laser are installed as common-use apparatus. The typical pulse energy at the focus is $\sim 90 \%$ of the pulse energy measured by GM1 in the wavelength range shorter than $30 \mathrm{~nm}$. We do not have an experimental chamber dedicated to the station at the moment, as most users are expected to bring their own UHV chambers.

2.3.1. KB mirror system. The specification of the KB mirror system at $L=85 \mathrm{~m}$ is summarized in Table 3 . The sizes of the mirrors are designed to be large enough to accept a beam size of $15 \mathrm{~mm}$, which is sufficiently larger than that restricted by the last orifice ( $6 \mathrm{~mm}$ diameter) of GM2.

Fig. 5 shows a focused beam profile at $\lambda=12.4 \mathrm{~nm}$ measured using knife-edge scanning of an Au wire (200 $\mu \mathrm{m}$ diameter). The spot size was $\sim 7.3 \mu \mathrm{m}$ (FWHM) in the horizontal and $\sim 10 \mu \mathrm{m}$ (FWHM) in the vertical. The slight difference between the horizontal and the vertical spot size is due to the difference of the focal lengths. A larger spot size is also available at the defocusing condition. For example, the spot size is enlarged to $\sim 30 \mu \mathrm{m}$ in the horizontal (FWHM) and $\sim 40 \mu \mathrm{m}$ in the vertical (FWHM) at a location $20 \mathrm{~mm}$ upstream of the focal position.

2.3.2. Synchronized optical laser system. The synchronized Ti:sapphire laser for pump-probe experiments is located in the laser hutch, which is about $13 \mathrm{~m}$ away from EH4a. The basic scheme of the laser system and the synchronization system is similar to that employed for BL2 and BL3 (Tono et 


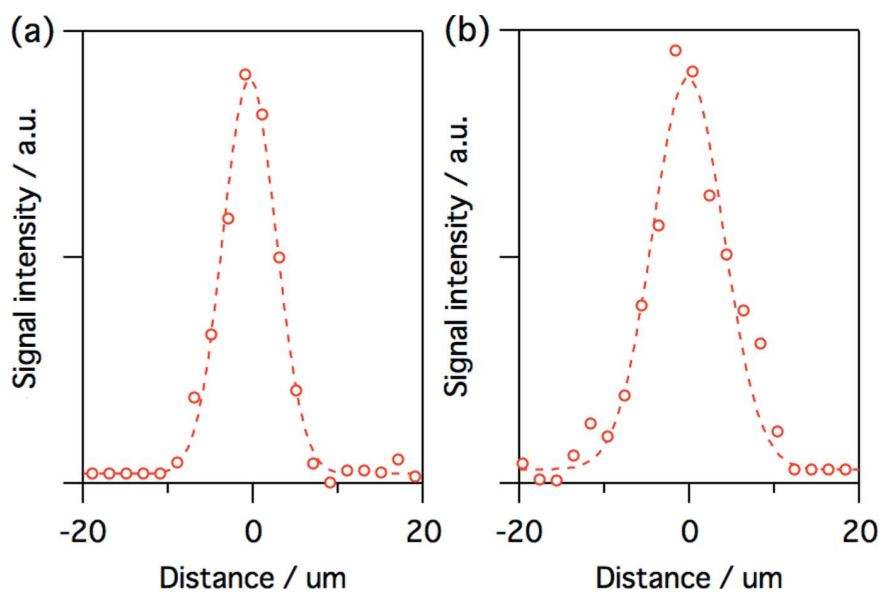

Figure 5

Spatial profiles of the focused beam with the KB mirror in the $(a)$ horizontal and $(b)$ vertical direction, measured at $\lambda=12.4 \mathrm{~nm}$ with the knife-edge scan method. The red circles are measured results, while the dashed lines are Gaussian fits. The knife-edge scan measurement was performed in the vacuum chamber using a gold wire, motorized stages and a silicon photodiode. The soft X-ray FEL pulse energy was reduced using the gas attenuator at an $\mathrm{N}_{2}$ pressure of $70 \mathrm{~Pa}$ combined with $1.1 \mu \mathrm{m}$ $\mathrm{Zr}$ foil to eliminate the contribution of the higher-order harmonics.

al., 2013). The laser system consists of a mode-locked oscillator (Vitara, Coherent Inc.), a chirped pulse amplification system (Legend Elite, Coherent Inc.) and a home-build multipass amplifier. This laser system generates a pulse energy of $\sim 10 \mathrm{~mJ}$ with a pulse duration of $\sim 40$ fs at $\lambda=800 \mathrm{~nm}$ after the pulse compression performed at the experimental station. The central wavelength in the range $200-2600 \mathrm{~nm}$ is available by combining the second, the third and the fourth harmonics of $800 \mathrm{~nm}$, and the optical parametric amplifier (HE-TOPAS Prime, Light Conversion).

The timing of optical laser pulses is synchronized to the master clock of the SACLA accelerator, which is commonly used to drive the SCSS+ accelerator. The cavity length in the

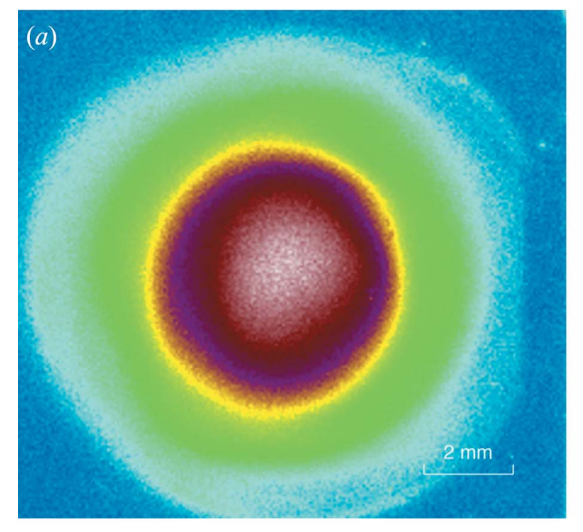

Figure 6

(a) Spatial profile of the FEL beam at SCM3 and $(b)$ shot-to-shot FEL spectra measured at $\lambda=$ $12.4 \mathrm{~nm}$.

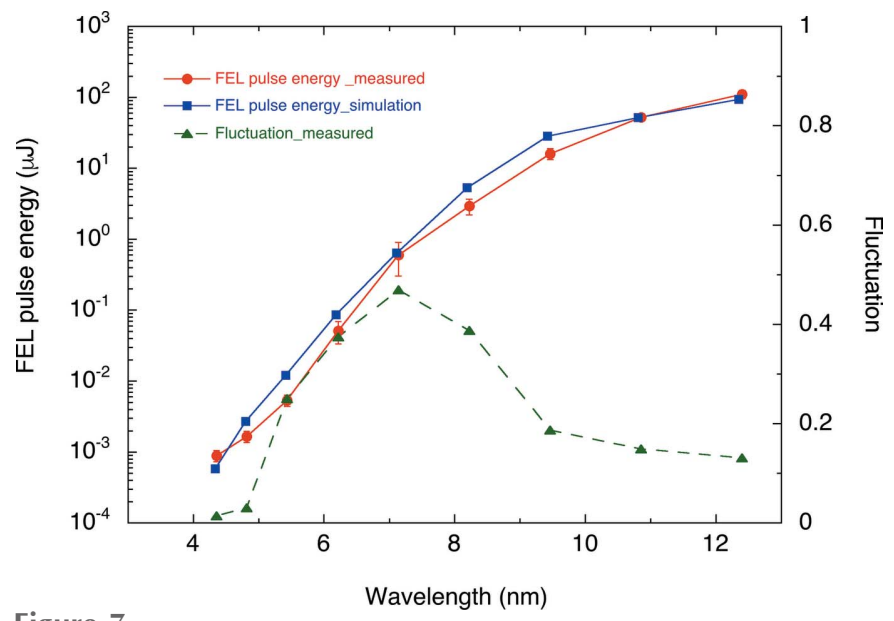

Figure 7

Averaged FEL pulse energy and its shot-by-shot fluctuation as a function of wavelength. The red solid circles are measured pulse energies while the blue circles are simulated ones. The green triangles are the measured fluctuation.

oscillator was compensated for synchronization (SynchrolockAP, Coherent Inc.). For the fine delay scan, a high-precision optical delay stage (custom-made, Kohzu Seiki) with a resolution of $6.7 \mathrm{fs}$ per pulse is installed in the experimental station. For coarse delay scans, a phase shifter (84DgR5B01, CANDOX systems) with a resolution of $\sim 1$ ps can be used.

\section{Performance}

The typical spatial profile at SCM3 $(L=42 \mathrm{~m})$ and shot-toshot spectra of soft X-ray FEL pulses at $\lambda=12.4 \mathrm{~nm}$ are shown in Fig. 6. We observed that the spatial profile was close to a symmetric Gaussian distribution. The spectral resolution was measured to be $\Delta \lambda / \lambda \simeq 2 \%$.

Fig. 7 shows the averaged FEL pulse energy measured as a function of the undulator $K$-value and radiation wavelength $\lambda$. The electron beam energy and the bunch charge for this measurement were $780 \mathrm{MeV}$ and $0.23 \mathrm{nC}$, respectively. The electron beam envelope and the accelerator parameters were optimized at $K=2.1$. At each $K$ value, the injection beam orbit to the undulator was adjusted to maximize the pulse energy. To cover a high dynamic range across the wide spectral region, we used different detector sets for the pulse energy measurement: a calorimeter and the gas intensity monitor for high pulse energy $(>1 \mu \mathrm{J})$ and a silicon photodiode with the gas attenuator at low pulse energy $(<1 \mu \mathrm{J})$. Since the iris with $6 \mathrm{~mm}$ aperture limits the transverse aperture to the detector, the measured pulse energies are reduced at long wavelengths. To obtain the pulse energies at the undulator, the measured values were corrected by the transmission ratio 
calculated from the FEL spatial distribution and the iris aperture.

The pulse-energy fluctuation at each $K$-value was also plotted in Fig. 7. As can be seen from this figure, the fluctuation at the shortest wavelength $\lambda=4.4 \mathrm{~nm}$ with $K=0.5$ was small, the same as the fluctuation of the bunch charge, because only spontaneous radiation was generated in this condition. With increasing $K$-value, the SASE process grew exponentially and the fluctuation reached a maximum of $47 \%$ at $\lambda=$ $7.1 \mathrm{~nm}$. The pulse energy continuously increased to $110 \mu \mathrm{J}$ while the fluctuation decreased to $13 \%$ at $\lambda=12.4 \mathrm{~nm}$; namely, the SASE FEL output was saturated around $10 \mathrm{~nm}$.

Using the measured dependence of the pulse energy on the undulator $K$-value, we estimated the properties of the electron beam combined with SIMPLEX. The result is shown in Fig. 7 together with the experimental data. The beam conditions assumed in the simulation were a Gaussian distribution with a peak current of $300 \mathrm{~A}$, a length of $0.7 \mathrm{ps}$ (FWHM) and a normalized root-mean-squared emittance of $0.5 \mathrm{~mm} \mathrm{mrad}$. The simulation based on these assumptions reproduces the experimental data fairly well. The tendency of slight overestimation of the simulated pulse energy could arise from an inaccuracy arising from the assumption of a longitudinal current profile of the electron beam. The actual current profile will be measured directly using an RF deflector or other devices in the future.

Since the SCSS+ accelerator does not have the function to correct second-order nonlinearity of the beam energy chirp, which is generated in the RF acceleration and the bunch compression processes, the peak current is restricted to be a few hundred amperes. In order to increase the FEL pulse energy, a harmonic RF cavity or multipole magnets are planned to be installed for the nonlinear correction.

\section{Conclusion and perspective}

As the third FEL beamline of SACLA, the soft X-ray FEL beamline BL1 that employs the dedicated accelerator SCSS+ has been successfully developed. The FEL pulse energy reached $110 \mu \mathrm{J}$ at $\lambda=12.4 \mathrm{~nm}$, which agreed with the simulated result. The KB focusing mirror system and the synchronized optical laser system are operated as common experimental infrastructures.

Based on these achievements, user operation of BL1 started in July 2016. In parallel to the operation, upgrades and developments of the beamline continue. For example, we plan to install an arrival-timing monitor between soft X-ray FEL and optical laser pulses (Maltezopoulos et al., 2008; Harmand et al., 2013; Katayama et al., 2016), and a nondestructive spectrometer (Frassetto et al., 2008; Brenner et al., 2011) at the experimental station. We will also expand the diameter of the orifices to $10 \mathrm{~mm}$ in the transport channel in order to improve the beam profile with increased transmission in long-wavelength regions.

Furthermore, extra space in the SACLA undulator hall is available for installing additional C-band accelerator units and increasing the electron beam energy to $\sim 1.7 \mathrm{GeV}$, which enables shortening of the radiation wavelength to below $\lambda=2.6 \mathrm{~nm}(h v=470 \mathrm{eV})$ at $K=2.1$. Finally, synchronized operation of soft X-ray FEL and hard X-ray FEL pulses can be performed, as the timing of the SCSS+ linac is synchronized with that of the SACLA linac.

\section{Acknowledgements}

The authors are grateful to the AIST group (Drs Takahiro Tanaka, Masahiro Kato, Norio Saito) for their help in measuring the absolute pulse energy of the soft X-ray FEL, and to Dr Ichiro Inoue, Dr Akira Kon and Dr Yuichi Inubushi for their help in calibration of the spectrometer. The authors also thank all the SACLA staff.

\section{References}

Ackermann, W. et al. (2007). Nat. Photon. 1, 336-342.

Allaria, E. et al. (2012). Nat. Photon. 6, 699-704.

Andruszkow, J. et al. (2000). Phys. Rev. Lett. 85, 3825-3829.

Bencivenga, F., Cucini, R., Capotondi, F., Battistoni, A., Mincigrucci, R., Giangrisostomi, E., Gessini, A., Manfredda, M., Nikolov, I. P., Pedersoli, E., Principi, E., Svetina, C., Parisse, P., Casolari, F., Danailov, M. B., Kiskinova, M. \& Masciovecchio, C. (2015). Nature (London), 520, 205-208.

Boutet, S. et al. (2012). Science, 337, 362-364.

Brenner, G., Kapitzki, S., Kuhlmann, M., Ploenjes, E., Noll, T., Siewert, F., Treusch, R., Tiedtke, K., Reininger, R., Roper, M. D., Bowler, M. A., Quinn, F. M. \& Feldhaus, J. (2011). Nucl. Instrum. Methods Phys. Res. A, 635, S99-S103.

Emma, P. et al. (2010). Nat. Photon. 4, 641-647.

Erk, B. et al. (2014). Science, 345, 288-291.

Frassetto, F., Cocco, D., Zangrando, M. \& Poletto, L. (2008). Nucl. Instrum. Methods Phys. Res. A, 593, 129-131.

Glover, T. E., Fritz, D. M., Cammarata, M., Allison, T. K., Coh, S., Feldkamp, J. M., Lemke, H., Zhu, D., Feng, Y., Coffee, R. N., Fuchs, M., Ghimire, S., Chen, J., Shwartz, S., Reis, D. A., Harris, S. E. \& Hastings, J. B. (2012). Nature (London), 488, 603-608.

Hara, T., Inubushi, Y., Katayama, T., Sato, T., Tanaka, H., Tanaka, T., Togashi, T., Togawa, K., Tono, K., Yabashi, M. \& Ishikawa, T. (2013). Nat. Commun. 4, 2919-2924.

Harmand, M., Coffee, R., Bionta, M. R., Chollet, M., French, D., Zhu, D., Fritz, D. M., Lemke, H. T., Medvedev, N., Ziaja, B., Toleikis, S. \& Cammarata, M. (2013). Nat. Photon. 7, 215-218.

Henke, B. L., Gullikson, E. M. \& Davis, J. C. (1993). At. Data Nucl. Data Tables, 54, 181-342.

Inagaki, T., Kondo, C., Maesaka, H., Ohshima, T., Otake, Y., Sakurai, T., Shirasawa, K. \& Shintake, T. (2014). Phys. Rev. ST Accel. Beams, 17, 080702.

Inoue, I., Inubushi, Y., Sato, T., Tono, K., Katayama, T., Kameshima, T., Ogawa, K., Togashi, T., Owada, S., Amemiya, Y., Tanaka, T., Hara, T. \& Yabashi, M. (2016). Proc. Natl Acad. Sci. USA, 113, 1492-1497.

Ishikawa, T. et al. (2012). Nat. Photon. 6, 540-544.

Katayama, T., Owada, S., Togashi, T., Ogawa, K., Karvinen, P., Vartiainen, I., Eronen, A., David, C., Sato, T., Nakajima, K., Joti, Y., Yumoto, H., Ohashi, H. \& Yabashi, M. (2016). Struct. Dyn. 3, 034301.

Kim, K. H. et al. (2015). Nature (London), 518, 385-389.

Lambert, G., Hara, T., Garzella, D., Tanikawa, T., Labat, M., Carre, B., Kitamura, H., Shintake, T., Bougeard, M., Inoue, S., Tanaka, Y., Salieres, P., Merdji, H., Chubar, O., Gobert, O., Tahara, K. \& Couprie, M. E. (2008). Nat. Phys. 4, 296-300.

Maltezopoulos, T., Cunovic, S., Wieland, M., Beye, M., Azima, A., Redlin, H., Krikunova, M., Kalms, R., Frühling, U., Budzyn, F., 
Wurth, W., Föhlisch, A. \& Drescher, M. (2008). New J. Phys. 10, 033026.

Mimura, H., Yumoto, H., Matsuyama, S., Koyama, T., Tono, K., Inubushi, Y., Togashi, T., Sato, T., Kim, J., Fukui, R., Sano, Y., Yabashi, M., Ohashi, H., Ishikawa, T. \& Yamauchi, K. (2014). Nat. Commun. 5, 3539.

Moshammer, R. et al. (2007). Phys. Rev. Lett. 98, 203001.

Nango, E. et al. (2016). Science, 354, 1552-1557.

Rohringer, N., Ryan, D., London, R. A., Purvis, M., Albert, F., Dunn, J., Bozek, J. D., Bostedt, C., Graf, A., Hill, R., Hau-Riege, S. P. \& Rocca, J. J. (2012). Nature (London), 481, 488-491.

Sakurai, T., Ego, H., Inagaki, T., Asaka, T., Suzuki, D., Miura, S. \& Otake, Y. (2017). Phys. Rev. Accel. Beams, 20, 042003.

Sato, T., Okino, T., Yamanouchi, K., Yagishita, A., Kannari, F., Yamakawa, K., Midorikawa, K., Nakano, H., Yabashi, M., Nagasono, M. \& Ishikawa, T. (2008). Appl. Phys. Lett. 92, 154103. Shintake, T. et al. (2008). Nat. Photon. 2, 555-559.

Shintake, T. et al. (2009). Phys. Rev. ST Accel. Beams, 12, 070701.

Shwartz, S., Fuchs, M., Hastings, J. B., Inubushi, Y., Ishikawa, T., Katayama, T., Reis, D. A., Sato, T., Tono, K., Yabashi, M., Yudovich, S. \& Harris, E. (2014). Phys. Rev. Lett. 112, 163901.

Tamasaku, K., Shigemasa, E., Inubushi, Y., Katayama, T., Sawada, K., Yumoto, H., Ohashi, H., Mimura, H., Yabashi, M., Yamauchi, K. \& Ishikawa, T. (2014). Nat. Photon. 8, 313-316.

Tanaka, T. (2015). J. Synchrotron Rad. 22, 1319-1326.

Tanaka, T., Kato, M., Saito, N., Tono, K., Yabashi, M. \& Ishikawa, T. (2015). Rev. Sci. Instrum. 86, 093104.
Tanaka, T., Kitamura, H. \& Seike, T. (2008). Proceedings of the 30th International Free Electron Laser Conference (FEL 2008), Gyeongju, Korea, 24-29 August 2008, pp. 371-373.

Togashi, T. et al. (2011). Opt. Express, 19, 317-324.

Togawa, K., Shintake, T., Inagaki, T., Onoe, K., Tanaka, T., Baba, H. \& Matsumoto, H. (2007). Phys. Rev. ST Accel. Beams, 10, 020703.

Tomizawa, H., Sato, T., Ogawa, K., Togawa, K., Tanaka, T., Hara, T., Yabashi, M., Tanaka, H., Ishikawa, T., Togashi, T., Matsubara, S., Okayasu, Y., Watanabe, T., Takahashi, E. J., Midorikawa, K., Aoyama, M., Yamakawa, K., Owada, S., Iwasaki, A. \& Yamanouchi, K. (2015). High Power Laser Sci. Eng. 3, e14.

Tono, K., Togashi, T., Inubushi, Y., Sato, T., Katayama, T., Ogawa, K., Ohashi, H., Kimura, H., Takahashi, S., Takeshita, K., Tomizawa, H., Goto, S., Ishikawa, T. \& Yabashi, M. (2013). New J. Phys. 15, 083035 .

Wang, D. X., Krafft, G. A. \& Sinclair, C. K. (1998). Phys. Rev. E, 57, 2283-2286.

Yabashi, M., Tanaka, H. \& Ishikawa, T. (2015). J. Synchrotron Rad. 22, 477-484.

Yoneda, H., Inubushi, Y., Nagamine, K., Michine, Y., Ohashi, H., Yumoto, H., Yamauchi, K., Mimura, H., Kitamura, H., Katayama, T., Ishikawa, T. \& Yabashi, M. (2015). Nature (London), 524, 446449.

Yoneda, H., Inubushi, Y., Yabashi, M., Katayama, T., Ishikawa, T., Ohashi, H., Yumoto, H., Yamauchi, K., Mimura, H. \& Kitamura, H. (2014). Nat. Commun. 5, 5080. 